\title{
Brazilian Symposium on Computing System Engineering
}

\author{
(November 3-7, 2014, Manaus, Brazil)
}

The Brazilian Symposium on Computing System Engineering (SBESC) is an initiative of the research community originally associated with three events: the Brazilian Workshop on Real-Time Systems, created in 1998; the Brazilian Workshop on Operating Systems, created in 2004; and the Brazilian Workshop on Embedded Systems, created in 2010. The identification of a strong synergy among these research areas added to the fact that designing computing systems is an increasingly multidisciplinary task and motivated the workshops to move from their native conferences to form an independent symposium. Last year, the symposium incorporated another related research community, which is focused on topics related to Critical Embedded Systems such as system safety and dependability, and promoted an Education Forum. This year, besides the Education Forum, we also had an Industrial Track.

The technical program spanned a whole week, from November 3 to 7, and was organized in a main track composed of peer-reviewed paper and poster sessions. Also, two co-located events took place during SBESC, the Embedded Systems School (ESSE) and the Intel Embedded Systems Competition, where the first offered four tutorials covering topics such as embedded systems design using the Intel Galileo platform, autonomous vehicle engineering, FPGA reliability, and design of cyber-physical systems.

From the 81 papers submitted to the symposium this year, the Technical Program Committee selected 44 full papers and 10 posters. The program was also enriched by keynote presentations from Herbert Bos (Vrije Universiteit Amsterdam), Nikil Dutt (UC Irvine), John Impagliazzo (Hofstra University), Tim Kelly (University of York), and Hermann Kopetz (Vienna University). Prof. Bos presented his work on botnet detection and finding vulnerabilities in program binaries through reverse engineering. Prof. Dutt talked about cyber-physical systems-on-chip (SoCs) that are able to adapt their behavior in response to varying environmental conditions. Prof. Impagliazzo discussed the evolution of computing curricula, with an emphasis on the forthcoming ACM/IEEE CE2016 Report on Computer Engineering curriculum. Prof. Kelly gave a talk on fundamental principles of software safety assurance and the methods and means to achieve them. Prof. Kopetz discussed the Time-Triggered Architecture, a computing infrastructure for the design and implementation of dependable distributed embedded systems.

The SBESC Technical Program Committee selected one paper from those presented at the symposium to appear in the Operating Systems Review and thus offer ACM fellows a glimpse of the event. The authors were invited to extend and improve their manuscript with their most recent research results. In their paper named "On the Influence of Shared Memory Contention in Real-time Multicore Applications", Givani Gracioli and Antônio Augusto Frölhich assess the influence of contention for shared data memory in the context of real-time multicore applications. They evaluated multicore processors with different cache-coherence protocols and memory architectures using a benchmark (also presented in the paper), and found out that memory contention can cause significant application slowdown.

We would like to thank everyone that contributed to make SBESC a high-quality forum for discussing important aspects of computing systems engineering, and invite OSR readers to join this exciting community in Foz do Iguaçu, home of the breathtaking Iguaçu Falls, where SBESC will be held in 2015.

Raimundo Barreto (UFAM) SBESC'14 General Chair
Rafael Obelheiro (UDESC)

Operating Systems Chair
Leandro Becker (UFSC)

SBC ESC SIG Chair 